\title{
Quantum plasmonics: Symmetry-dependent plasmon-molecule coupling and quantized photoconductances
}

\author{
Peng Song, ${ }_{1}^{1}$ Sheng Meng, ${ }^{1}$ Peter Nordlander, ${ }^{2}$ and Shiwu Gao ${ }^{3, *}$ \\ ${ }^{1}$ Beijing National Laboratory for Condensed Matter Physics, and Institute of Physics, Chinese Academy of Sciences, 100190, Beijing, China \\ ${ }^{2}$ Department of Physics, Rice University, Houston, Texas 77005, USA \\ ${ }^{3}$ Department of Physics, University of Gothenburg, SE-41296, Gothenburg, Sweden
}

(Received 12 June 2012; published 26 September 2012)

\begin{abstract}
We investigate photoinduced electron transport through nanoparticle dimers connected by linear and ringlike molecules. The two model junctions exhibit similar absorption but distinct photoconductance. The linear-chain junction shows a constant conductance that is independent of photon energies. In contrast, the ring molecules show quantized conductance resonances at energies corresponding to the molecular excitations. These results can be understood from the symmetry and quantization of local molecular excitations, and have implications for plasmon-molecule interactions in general.
\end{abstract}

DOI: 10.1103/PhysRevB.86.121410

PACS number(s): 73.20.Mf, 71.45.Gm, 72.15.Nj

Light-induced electronic excitation and its coupling to electron transport in a molecular junction play central roles in energy harvesting applications such as solar cells, ${ }^{1,2}$ photocatalysis, ${ }^{3-5}$ and optoelectronic devices. ${ }^{6-8}$ Various artificial metallic nanostructures have been fabricated to optimize the absorption and the efficiency of photoenergy conversion. ${ }^{9-11}$ The optical responses of metallic nanostructures are generally dominated by their localized surface plasmon resonances (LSPRs), whose nonradiative relaxation generates hot carriers. In a tunnel junction, these hot carriers can participate and interplay with electron transport. Recently, plasmonic enhancement of photocurrents has been detected in metallic junctions consisting of a few atoms, ${ }^{12,13}$ molecular wires, ${ }^{14,15}$ and thin films loaded with gold nanoparticles. ${ }^{1,5}$ The frequency dependence of the photocurrents has been associated with the surface plasmons of the systems. However, the mechanism and dynamics of plasmon-enhanced photocurrents are not well understood, because many intermediate steps involving plasmon excitation, plasmon decay into hot carriers, and its coupling to electron transport are not accessible experimentally. Theoretical investigations of plasmonmolecule coupling are therefore of paramount importance for the understanding and control of these processes.

Here we present a fully quantum-mechanical study of plasmon excitation and electron transport across molecular junctions consisting of linear and ringlike atomic chains, which form serial and parallel connections between two nearby metallic nanoparticles (NPs, see the inset of Fig. 1). Similar prototype junctions have been realized experimentally by a scanning tunneling microscope or nanofabrication..$^{12-18}$ In accordance with experimental findings, all these junctions exhibit enhanced photocurrents at the plasmon resonances, whose intensities depend sensitively on the symmetry of molecular structures. For the chain connections, the coupling to longitudinal molecular excitations is dominant, leading to a nearly constant conductance. In contrast, for the ring connections, the coupling to the out-of-plane excitations dominates and results in conductance resonances with intensities that are proportional to the number of atoms. These features can be understood from the symmetry and quantization of the localized molecular excitations and their coupling to the surrounding metal electrodes.
All calculations have been performed with the real-space time-dependent density functional theory (TDDFT) code, OCTOPUS. ${ }^{19-22}$ The structures of the molecular junctions are sketched in the inset of Fig. 1, where two nanoparticles with a diameter of $2.54 \mathrm{~nm}$ are connected by either a ring molecule (a) or a linear atomic chain (b). The separation between the molecule and both NPs is kept at $4 \AA$, which represents a typical contact distance. ${ }^{22}$ While the distances between the $\mathrm{Na}$ atoms are kept at $3.72 \AA$ corresponding to the interatomic distances in $\mathrm{Na}$ metal. The unit cell is generated with the minimum-cell scheme by assigning a sphere of $22 \AA$ in radius to each NP and atom in the system. The grid size is 0.5 $\AA$ and the convergence is set to $1.0 \times 10^{-3}$ in relative charge density. The conduction electrons of the NPs are described using the jellium approximation with an electron density $r_{\mathrm{s}}=3.0$ a.u. that simulates Ag NPs without the polarizability of the positive background charges. ${ }^{22,23}$ All Na atoms are described by the Troullier-Martins pseudopotentials ${ }^{24}$ in the local density approximation (LDA). ${ }^{25}$ For excitation, the system was perturbed by a $\delta$-function impulse at $t=0$, and subsequently evolved for 8000 steps with a time step of $0.003 \mathrm{fs}$. The timedependent electric current and voltage across the gap were Fourier transformed to yield the frequency-dependent current $I(\omega)$ and voltage $U(\omega)$, whose ratio defines a dynamical conductance $G(\omega)=I(\omega) / U(\omega)$ of the molecular junctions quantum mechanically. ${ }^{22}$

When conductive contact is established between two adjacent nanostructures, the optical properties will change. ${ }^{26-34}$ The effects of conducting junctions on the optical response of a plasmonic NP homodimer have been studied previously using classical electromagnetic modeling and assuming energyindependent junction conductances. ${ }^{28}$ These studies show that for small conductances, the optical spectra are dominated by a hybridized bonding dipolar plasmon (BDP) mode. Such classical studies give no information on the plasmon-molecule coupling at the molecular scale. The present work represents a significant advance in the study of plasmon-induced photoconductance in the quantum regime, where molecular electronic structures are treated explicitly and all electronic responses are calculated within a fully quantum-mechanical approach.

Figure 1 shows the absorption spectra of the dimer junctions consisting of $N=1-6$ sodium atoms in the ring (a) and chain 


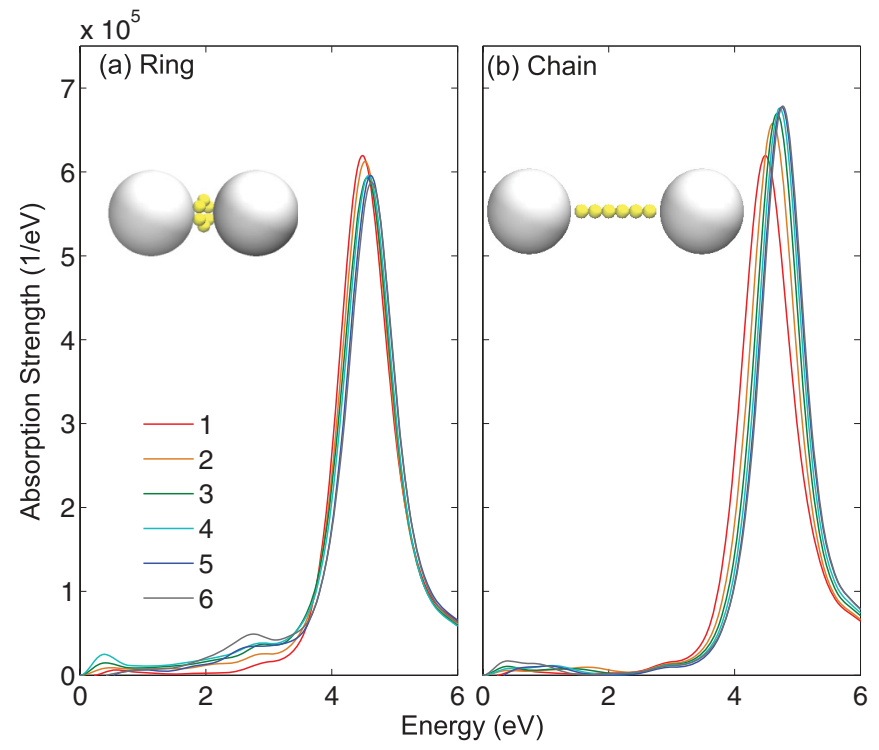

FIG. 1. (Color online) Absorption spectra of the ring (a) and chain (b) connected junctions of a nanoparticle dimer with a $2.54 \mathrm{~nm}$ diameter. The number of atoms in the molecules ranges from $N=1$ to 6. Three collective modes are visible: the CTP around $0.5 \mathrm{eV}, \mathrm{ME}$ around $1-3 \mathrm{eV}$, and SBDP around $4.6 \mathrm{eV}$.

(b) configurations. As a common feature, all curves show a dominant peak at around $4.6 \mathrm{eV}$, which is the hybridized BDP mode that also presents in classical calculations. ${ }^{27}$ For the ringlike molecules in Fig. 1(a), the energy of the BDP mode blueshifts from 4.53 to $4.63 \mathrm{eV}$ as $N$ increases from 1 to 6 . This blueshift is accompanied by a reduced intensity and a broadening in line shape. These changes are characteristics of the formation of the screened BDP mode (SBDP) ${ }^{28}$ and result from the increased coupling of the molecules to the plasmon mode due to the addition of atoms in the gap. Note that the direct electromagnetic coupling between the NPs is essentially unchanged since the gap width is fixed at $8 \AA$. In the classical model, the blueshift is interpreted by the reduced capacitive coupling between the NPs when the junction is filled by a conductive medium and the broadening is due to the increased dissipation caused by electron transport across the junction. ${ }^{28}$ In a quantum-mechanical picture, the addition of electrons in the gap due to the presence of atom rings is responsible for the enhanced coupling between the NPs, leading to the blueshift of the BDP mode. Its broadening is caused by the addition of more electronic channels for the plasmon decay with increasing $N$. In addition to the BDP mode, two smaller peaks, one at $0.5 \mathrm{eV}$ and another at $2.5 \mathrm{eV}$, also develop for larger rings. The $0.5 \mathrm{eV}$ mode is the charge transfer plasmon (CTP) mode resulting from electron tunneling between the two NPs. ${ }^{22,28,35}$ The $2.5 \mathrm{eV}$ mode is associated with molecular excitation (ME), which is the main focus of the current work. As we see below, these $\mathrm{ME}$ resonances reveal the coupling mechanism between the plasmon excitations and local electronic excitations of the molecules.

For the chain junctions shown in Fig. 1(b), the energy of the BDP mode also shows a blueshift from 4.53 to $4.67 \mathrm{eV}$ as the chain length increases from $N=1$ to 6 . However, its intensity increases with $N$ and its linewidth decreases monotonically.

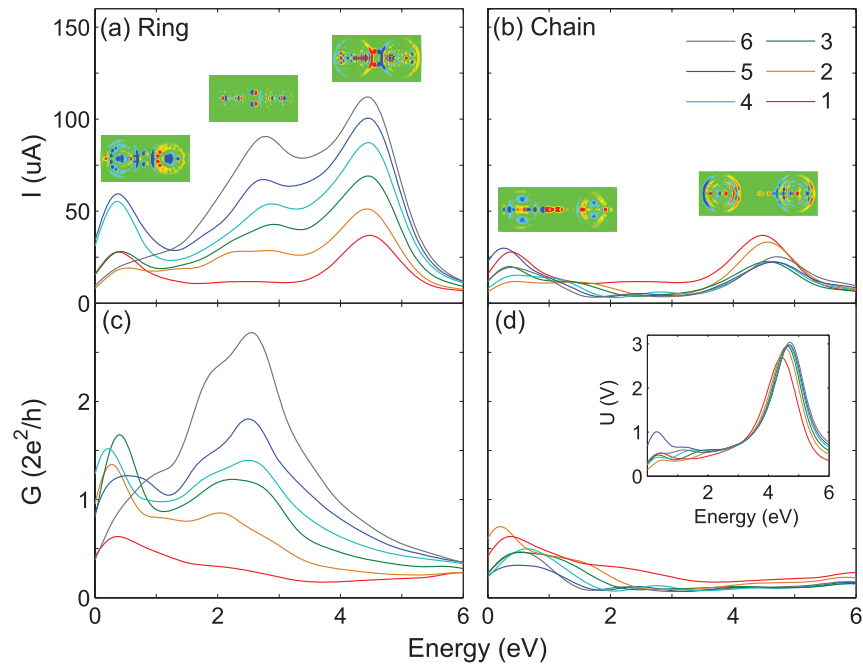

FIG. 2. (Color online) Photocurrent (upper panels) and photoconductance (lower panels) spectra of the ring (left panels) and chain (right panels) junctions. The currents and conductances of rings increase in a steplike fashion with the increasing number of atoms. The insets in (a) and (b) show the distinct charge density excitations at the plasmon resonance peaks.

Both the blueshift and decreased linewidth are characteristics of the reduced hybridization of the BDP mode, as the gap becomes wider. Similar gap-width dependence of the BDP mode was also observed in bare NP dimers ${ }^{23}$ and in single atom junctions. ${ }^{22}$ Apart from the BDP resonance, there is also a weak CTP mode at $0.5-1 \mathrm{eV}$. Unlike the ring junctions, there is no sign of a ME resonance for the chain-connected junctions. This difference reflects the sensitivity of optical excitation to the geometry and electronic structure of the molecular junctions.

Figure 2 shows the frequency-dependent photocurrents [(a) and (b)] and photoconductances [(c) and (d)] for the chain and ring junctions. In both cases, the photocurrents exhibit similar resonances as the absorption curves in Fig. 1. Obviously, there is a general correlation between plasmon excitation and photocurrents for the conductive junctions. This relation is particularly prominent for the ring connections. It is also interesting to note that the BDP mode does not show up strongly in either of the photoconductance spectra and that no $\mathrm{ME}$ resonance is present for the chain junctions. The insets show the induced charge distributions of the corresponding resonance peaks.

As shown in Fig. 2(a), the photocurrent spectra for the ring junctions exhibit two prominent peaks: the BDP at $4.6 \mathrm{eV}$ and the ME at $2.7 \mathrm{eV}$. The intensities of both peaks increase almost linearly with $N$. For example, the current at $4.6 \mathrm{eV}$ goes from $30 \mu \mathrm{A}$ for $N=1$ to $120 \mu \mathrm{A}$ for $N=6$, giving a fourfold increase. For the $\mathrm{ME}$ at $2.7 \mathrm{eV}$, the photocurrent is enhanced from 10 to $85 \mu \mathrm{A}$ with an eightfold increase. At both peaks, the photocurrents exhibit stepwise increase as a function of $N$. This behavior is similar to dc transport, where the dc conductance often shows steplike function with the number of channels involved in the transport.

The photocurrents for the chains in Fig. 2(b) are generally much smaller than for the rings and linger around 5-10 $\mu \mathrm{A}$ 
over the whole 1-3 eV region. Like the ring connections, they also show resonant peaks at the BDP and CTP modes. However, their intensities do not exhibit a monotonous increase with increasing number of atoms. Instead, the photocurrent at the BDP mode decreases slightly when $N$ increases from 1 to 3 , and then remains nearly constant for $N>3$. As another drastic difference between the rings and chains, there is no $\mathrm{ME}$ resonance for the chain junctions.

Although the photocurrents in Fig. 2(a) increase linearly at both the BDP and ME modes, the enhancement mechanisms are different. The current increase at the BDP mode is mainly associated with the enhanced electric field of the plasmon excitations, which in turn leads to an enhanced voltage across the gap as shown in the inset of Fig. 2(d). The simultaneous increase in current and voltage gives thus a relatively featureless conductance in this region as shown in Fig. 2(c). In contrast, the voltages at the $\mathrm{ME}$ resonance around $2.7 \mathrm{eV}$ are relatively constant, the photocurrent increase is therefore not caused by the voltage but rather by plasmon-enhanced molecular excitations. Such local excitations contribute to the intrinsic photoconductance of the molecules. ${ }^{36}$ In comparison, the conductance spectra of Fig. 2(d) at 4.6 and $2.7 \mathrm{eV}$ are flat and featureless. The increased photocurrent at the BDP mode is also due to a plasmonic effect as in the ring cases.

The conductance of the CTP resonance exhibits oscillatory behavior with $N$ for both chain and ring connections. Such a conductance variation is consistent with what was observed for dc transport in linear sodium wires, which display even-odd oscillations as a function of the number of Na atoms. ${ }^{36}$ Indeed, our detailed analysis of the molecular orbitals also confirms that this oscillation is directly correlated to the quantization of their orbital energies and the change of occupancies, as the number of atoms is varied. It is relatively insensitive to the specific atomic configurations of the connection.

The dramatic differences between the photocurrents shown in Figs. 2(a) and 2(b) suggest that molecular geometry and local electronic structure play an important role in the photoinduced transport across the junctions. To gain insight into the mechanism of the plasmon-molecule coupling and its effect on photoconduction, we calculated the absorption spectra of the isolated rings and chains, which are shown in Fig. 3. The atoms are fixed at the same relative positions as in the junctions. For the linear atomic chains, there is a longitudinal (L) and two transverse $(\mathrm{T})$ absorption resonances corresponding to ME modes. ${ }^{20,21}$ The dipole excitation of the $\mathrm{L}$ mode is parallel to the symmetry axis across the gap and can couple to the SBDP mode. The energy of the $\mathrm{L}$ mode is around 1-2 eV, which is well below the frequency of the SBDP. Due to this energy mismatch, the linear chains only couple very weakly to the SBDP mode. This weak coupling explains the small photocurrents in Fig. 2(b) and the decreasing currents as the chain gets longer: as the chain gets longer, the $\mathrm{L}$ mode redshifts away from the SBDP and becomes more decoupled. The $\mathrm{T}$ mode has comparable energy with SBDP but does not match in symmetry and thus does not contribute to the photocurrent across the gap.

Similarly, atomic rings also have two types of excitations: one is polarized in the azimuthal plane of the rings (A) and another one is polarized perpendicularly to the ring plane (P). For the ring aligned in Fig. 1(a), only the $\mathrm{P}$ mode can
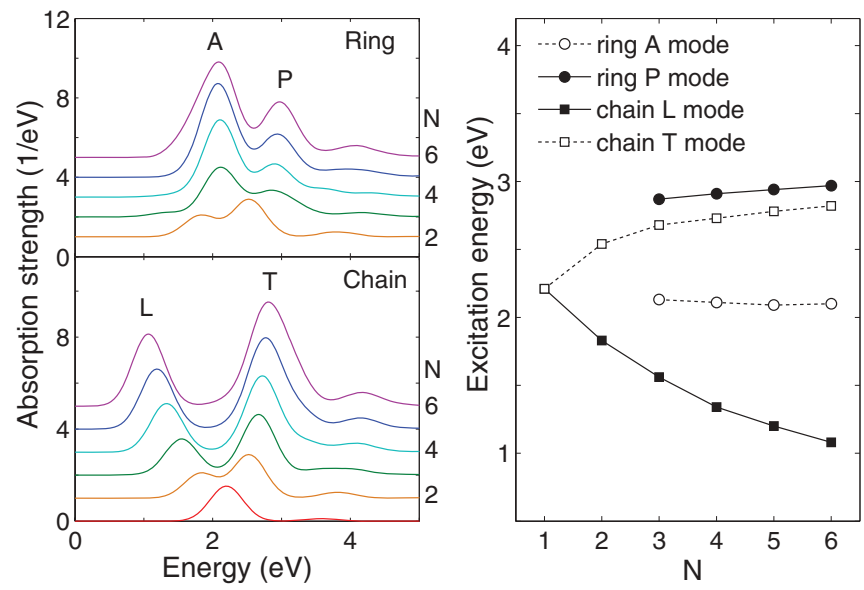

FIG. 3. (Color online) Absorption spectra (left panels) of isolated rings and chains as a function of the number of atoms. For rings, the A mode refers to polarization in the azimuthal plane of the rings and the $\mathrm{P}$ mode is for polarization perpendicular to the atomic plane. For the chains, the $\mathrm{L}$ mode is polarized along the chain while the $\mathrm{T}$ mode is polarized perpendicularly. The right panel shows the excitation energies of these modes. Only the two modes shown with the solid line can couple to the longitudinal dimer plasmons.

couple to the electric field in the junction. The energy of the $\mathrm{P}$ mode for the isolated molecules is $2-3 \mathrm{eV}$, which is comparable to the energy of the SBDP. The coupling between the molecular P mode and the SBDP is responsible for the enhanced photocurrents across the junction. The intensity of the $\mathrm{P}$ mode increases linearly with the number of atoms in the rings, which explains the linear increase of the photocurrents for both the ME mode around $2.7 \mathrm{eV}$ and the BDP mode around $4.6 \mathrm{eV}$. The results in Figs. 2 and 3 clearly show that the photocurrents of the molecular junctions are determined by two factors: (i) the match of symmetry between the molecular excitation and the plasmon mode of the cavity and (ii) the coupling strengths between them. For efficient coupling, a matched symmetry and comparable energies between the molecular excitations and the surface plasmons are required.

To further elaborate the coupling mechanisms outlined above, we analyzed the energy spectra and the symmetries of the molecular orbitals (MOs) for the electron-hole (e-h) pairs that are involved in the plasmon resonances. Figure 4 shows the local density of states (LDOS) of the ring (a) and chain (b) molecules for different $N$ in the junctions of a smaller NP dimer with a $1.69 \mathrm{~nm}$ diameter. For simplicity, only the LDOS between -1 and $2 \mathrm{eV}$ around the Fermi level is shown. For the rings, the unoccupied bands of the molecules can be divided approximately into two regions: one between $[0: 1] \mathrm{eV}$; and the other between $[1: 2.5] \mathrm{eV}$, which correspond to the $\sigma$ and $\pi$ bands of $3 p$ electrons, respectively. The $\mathrm{P}$ mode involves mainly the $s \rightarrow p_{z}$ transitions along the gap direction. Such transitions that match both the symmetry and energy of the $\mathrm{P}$ mode can all be identified, and the wave functions of a typical $\mathrm{e}-\mathrm{h}$ pair state are plotted in the inset of the upper panel. A similar analysis was also carried out for the chain connections. The LDOS shown in the lower panel shows the evolution of the band structures with different MO symmetries. The L mode excitation results from e-h pairs in the energy range between 


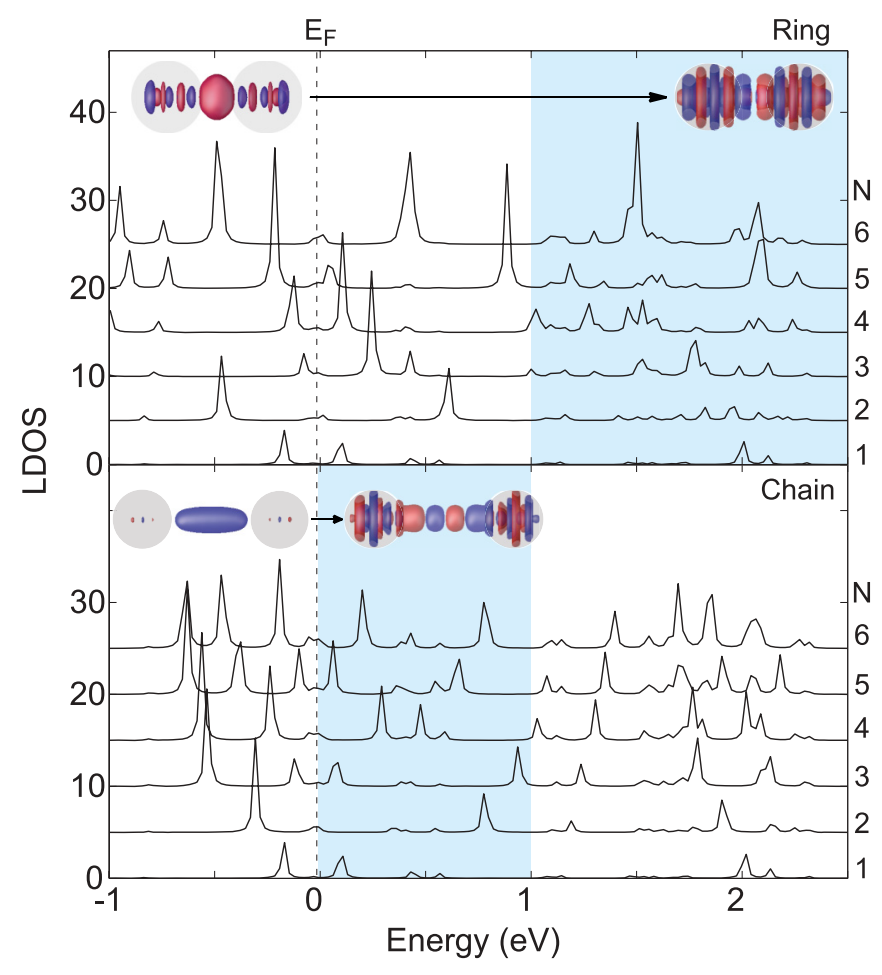

FIG. 4. (Color online) Local density of states of the ring and chain molecules for different $N$. The cyan background in the upper panel marks the final states involved in the P mode. In the lower panel, the cyan background is the corresponding region of the $\mathrm{L}$ mode for the chains. The insets show the wave functions of characteristic electron-hole pair states involved in these modes.

-1 and $1 \mathrm{eV}$ around the Fermi level. The orbitals of these e-h pairs are also characteristic for the $s \rightarrow p_{z}$ transitions as shown in the inset. The energy bands between 1 and $2.5 \mathrm{eV}$ correspond to the $p_{x}$ and $p_{y}$ orbitals perpendicular to the chains, which participate only in the $\mathrm{T}$ mode excitations. These states do not match the symmetry of the SBDP, and thus do not contribute to photocurrents.

In conclusion, we have investigated the coupling of plasmon excitation to electron transport through molecules in a plasmonic dimer junction. The results show that both the optical spectra and the photocurrents depend strongly on the symmetries and relative energies between the molecular excitations and the plasmon resonances. In general, the photoconductances of the molecules can exhibit strong resonance behaviors with molecular excitations of the right symmetry. Our findings are the following. (i) Moleculeplasmon coupling is dominated by the matched symmetry between the molecular excitations and the plasmons. (ii) Their relative energies also affect the strength of coupling, where the resonant condition favors strong coupling. We observe quantized photoconductance in the strong coupling regime for the parallel connections. This quantized conductance is similar to the DC conductance, but has different physical origin. These findings, though obtained for model junctions, are likely to hold for other molecular junctions in serial and parallel connections and are therefore of significant importance for the development of plasmon-enhanced photoconductance, solar cells, and optoelectronic applications.

We acknowledge financial supports from NSFC (Grant No. 11074287), MOST (2012CB921403), and hundred-talent program of CAS. P.N. acknowledges support from the Robert A. Welch Foundation (C-1222) and the Office of Naval Research (N000244-09-1-0989). S.G. acknowledges supports from the Foundation for Strategic Research (SSF) and the Institutional Grant Program of STINT. *shiwu.gao@physics.gu.se

${ }^{1}$ H. A. Atwater and A. Polman, Nat. Mater. 9, 205 (2010).

${ }^{2}$ F. Wang and N. A. Melosh, Nano Lett. 11, 5426 (2011).

${ }^{3}$ S. Linic, P. Christopher, and D. B. Ingram, Nat. Mater. 10, 911 (2011).

${ }^{4}$ P. Christopher, H. Xin, and S. Linic, Nat. Chem. 3, 467 (2011).

${ }^{5}$ Z. W. Liu, W. B. Hou, P. Pavaskar, M. Aykol, and S. B. Cronin, Nano Lett. 11, 1111 (2011).

${ }^{6}$ P. Banerjee, D. Conklin, S. Nanayakkara, T. H. Park, M. J. Therien, and D. A. Bonnell, ACS Nano 4, 1019 (2010).

${ }^{7}$ R. Arielly, A. Ofarim, G. Noy, and Y. Selzer, Nano Lett. 11, 2968 (2011).

${ }^{8}$ M. W. Knight, H. Sobhani, P. Nordlander, and N. J. Halas, Science 332, 702 (2011).

${ }^{9}$ N. Liu and H. Giessen, Angew. Chem. Int. Ed. 49, 9838 (2010).

${ }^{10}$ V. Giannini, A. I. Fernandez-Dominguez, S. C. Heck, and S. A. Maier, Chem. Rev. 111, 3888 (2011).

${ }^{11}$ S. M. Morton, D. W. Silverstein, and L. Jensen, Chem. Rev. 111, 3962 (2011).

${ }^{12}$ D. C. Guhr, D. Rettinger, J. Boneberg, A. Erbe, P. Leiderer, and E. Scheer, Phys. Rev. Lett. 99, 086801 (2007).

${ }^{13}$ N. Ittah, G. Noy, I. Yutsis, and Y. Selzer, Nano Lett. 9, 1615 (2009).
${ }^{14}$ A. I. Yanson, G. R. Bollinger, H. E. van den Brom, N. Agraït, and J. M. van Ruitenbeek, Nature 395, 780 (1997).

${ }^{15}$ R. F. Oulton, V. J. Sorger, T. Zentgraf, R. M. Ma, C. Gladden, L. Dai, G. Bartal, and X. Zhang, Nature 461, 629 (2009).

${ }^{16}$ T. M. Wallis, N. Nilius, and W. Ho, Phys. Rev. Lett. 89, 236802 (2002).

${ }^{17}$ C. Chen, C. A. Bobisch, and W. Ho, Science 325, 5943 (2009).

${ }^{18}$ H. Ohnishi, Y. Kondo, and K. Takayanagi, Nature 395, 780 (1998).

${ }^{19}$ M. A. L. Marques, A. Castro, G. F. Bertsch, and A. Rubio, Comput. Phys. Commun. 151, 60 (2003).

${ }^{20}$ J. Yan, Z. Yuan, and S. W. Gao, Phys. Rev. Lett. 98, 216602 (2007).

${ }^{21}$ J. Yan and S. W. Gao, Phys. Rev. B 78, 235413 (2008).

${ }^{22}$ P. Song, P. Nordlander, and S. W. Gao, J. Chem. Phys. 134, 074701 (2011).

${ }^{23}$ J. Zuloaga, E. Prodan, and P. Nordlander, Nano Lett. 9, 887 (2009).

${ }^{24}$ N. Troullier and J. L. Martins, Phys. Rev. B 43, 1993 (1991).

${ }^{25}$ D. M. Ceperley and B. J. Alder, Phys. Rev. Lett. 45, 566 (1980).

${ }^{26}$ T. Atay, J. H. Song, and A. V. Nurmikko, Nano Lett. 4, 1627 (2004).

${ }^{27}$ J. B. Lassiter, J. Aizpurua, L. I. Hernandez, D. W. Brandl, I. Romero, S. Lal, J. H. Hafner, P. Nordlander, and N. J. Halas, Nano Lett. 8 , 1212 (2008). 
${ }^{28}$ O. Perez-Gonzalez, N. Zabala, A. G. Borisov, N. J. Halas, P. Nordlander, and J. Aizpurua, Nano Lett. 10, 3090 (2010).

${ }^{29}$ N. Large, M. Abb, J. Aizpurua, and O. L. Muskens, Nano Lett. 10, 1741 (2010).

${ }^{30}$ A. I. Fernandez-Dominguez, S. A. Maier, and J. B. Pendry, Phys. Rev. Lett. 105, 266807 (2010).

${ }^{31}$ N. Berkovitch and M. Orenstein, Nano Lett. 11, 2079 (2011).
${ }^{32}$ O. Perez-Gonzalez, N. Zabala, and J. Aizpurua, New J. Phys. 13, 083013 (2011).

${ }^{33}$ D. Y. Lei, A. Aubry, Y. Luo, S. A. Maier, and J. B. Pendry, ACS Nano 5, 597 (2011).

${ }^{34}$ M. Hentshel, D. Dregely, R. Vogelgesang, H. Giessen, and N. Liu, ACS Nano 5, 2042 (2011).

${ }^{35}$ R. Esteban, A. G. Borisov, P. Nordlander, and J. Aizpurua, Nat. Commun. 3, 825 (2012).

${ }^{36}$ P. A. Khomyakov and G. Brocks, Phys. Rev. B 74, 165416 (2006). 